\title{
Functional Mitochondrial Heterogeneity in Heteroplasmic Cells Carrying the Mitochondrial DNA Mutation Associated with the MELAS Syndrome (Mitochondrial Encephalopathy, Lactic Acidosis, and Strokelike Episodes)
}

\author{
ANNETTE BAKKER, CYRILLE BARTHÉLÉMY, PAULE FRACHON, DANIELLE CHATEAU, \\ DAMIEN STERNBERG, JEAN PIERRE MAZAT, AND ANNE LOMBÈS
}

INSERM UR523, Institut de Myologie, 75651 Paris, France [A.B., C.B., P.F., D.C., A.L.]; Biochimie B, Hôpital de La Salpêtrière, 75651 Paris, France [D.S.]; and Université de Bordeaux II, INSERM E99-29, 33076 Bordeaux cedex, France [J.P.M.]

\begin{abstract}
ABST
Most mitochondrial DNA (mtDNA) alterations associated
with human disorders are heteroplasmic, i.e. mutant mtDNA
molecules coexist with normal ones within the cell. We ad-
dressed the possibility of intermitochondrial exchanges through
histologic analyses of cybrid clones with increasing proportion of
the MELAS (A3243G) mtDNA transfer RNA point mutation.
MtDNA-dependent cytochrome $c$ oxidase activity and protein
composition as well as mitochondrial membrane potential ap-
peared heterogeneous in individual cells from clonal heteroplas-
mic cell populations on the basis of confocal and electron
microscopy. The number of defective cells increased with in-
creasing mutation load. We conclude that in the presence of a
heteroplasmic mtDNA mutation in the cell type that we studied,
intermitochondrial molecular exchanges cannot provide an effi-
cient even distribution of the complementing molecules such as
\end{abstract}
Mitochondria are unique organelles in their double genetic origin. The vast majority of their proteins are encoded by nuclear DNA, but 13 essential subunits of the respiratory chain are encoded on mtDNA and transcribed and translated within mitochondria (1). Since 1988, numerous mtDNA alterations (deletions, duplications, and point mutations) have been described in association with very diverse human disorders (2). Most of these mtDNA alterations are heteroplasmic, that is, mutant mtDNA molecules coexist with normal ones leading to

Received September 7, 1999; accepted January 5, 2000.

Correspondence and reprint requests: Anne Lombès, M.D., INSERM UR 523, Institut de Myologie, Hôpital de La Salpêtrière, 75651 Paris cedex 13, France

Supported by a grant from the AFM (Association Française contre les Myopathies); A.B. was the recipient of an AFM fellowship. wild-type mtDNA, transfer RNA, or protein. Mitochondria in these heteroplasmic cells cannot, therefore, be considered a single functional unit. (Pediatr Res 48: 143-150, 2000)

\section{Abbreviations}

MELAS, mitochondrial myopathy, encephalopathy, lactic acidosis, and strokelike episodes

mtDNA, mitochondrial DNA

COX, cytochrome $c$ oxidase

tRNA, transfer RNA

rho0 cells, cells that are totally depleted of their mtDNA

rho+ cells, cells that have normal mtDNA

rho- cells, cells that have mtDNA carrying a large-size deletion the possibility of direct complementation between wild-type and mutant mtDNA molecules (3).

Cybrid clones are cells obtained by fusion between human rho0 cell lines (HeLa or osteosarcoma cells totally depleted of mtDNA) and enucleated cells from patients with an mtDNA alteration. They provide valuable tools for investigation of mtDNA defects excluding the influence of nuclear DNAencoded factors. Direct complementation between wild-type and mutant mtDNA molecules was demonstrated in such cybrid cells by the translation of an abnormal peptide encoded on mutant mtDNA through use of wild-type mtDNA tRNA $(4,5)$. However, whether the complementation events could occur only in heteroplasmic mitochondria or also between mitochondria was still highly debated. It led to the proposals of two 
almost opposite models of mitochondrial compartment. In one of them, each mitochondrion is functionally independent and exchanges very little, if any, genetic information with other mitochondria of the same cell. This model was favored by the segregation behavior of mtDNA in hybrid and cybrid models (6) and in successive generations of mammals (7). It was also supported by the absence of direct complementation between two tRNA point mutations introduced separately in the same cybrid clones, contrasting with the extensive direct complementation between wild-type and mutant mtDNA when both mtDNA species coexisted since the mutation event (8). On the other hand, a mitochondrial compartment with efficient molecular exchanges between mitochondria was deduced from wildtype mtDNA complementation by chloramphenicol-resistant mtDNA in hybrid and cybrid models (9), and by rapid diffusion of ethidium bromide-stained mtDNA to the whole mitochondrial network in cybrids between rho0 and rho + HeLa cells (10). This was recently reinforced by the occurrence of complementation between rho- and rho+/chloramphenicolresistant mtDNA (5) or between two different pathogenic mutant mtDNA (11) that were, in both experiments, introduced separately into the same cybrids.

Clinical status of human disorders associated with heteroplasmic mtDNA mutations is greatly dependent on the residual amount of wild-type mtDNA molecules. No clinical symptom or biochemical respiratory chain defect is detected above a relatively low threshold of wild-type mtDNA proportion (12). Therapeutic approaches to human disorders are, therefore, trying to modify the relative proportion of wild-type and mutant mtDNA (13). In that prospect, the real efficiency of intermitochondrial molecular exchanges is an essential parameter to establish because it will influence the fate of any induced increase of wild-type mtDNA. If mitochondria exchanged very little with each other, mitochondrial functions would be heterogeneous within a heteroplasmic cell. Random cytoplasmic partition of the cell mitochondrial population during mitoses would lead to heterogeneous cells with respect to their mitochondrial respiratory function. Any small induced change in the wild-type mtDNA proportion would, therefore, rapidly be segregated. On the other hand, if wild-type and mutated mtDNA molecules distributed evenly throughout a mitochondrial compartment behaving as a single dynamic unit, mitochondrial respiratory function would be homogeneous within each cell. Any induced increase in wild-type mtDNA proportion would be distributed to the whole cell, transmitted equally to daughter cells and maintained through successive mitoses.

We addressed the dynamics of intermitochondrial exchanges by the analysis of the functional behavior of heteroplasmic cells. We used histologic techniques because this strategy allowed us to study individual cells and to reach subcellular level with confocal and electron microscopy. Complementation was analyzed through the restoration of mtDNAdependent parameters such as COX (EC 1.9.3.1) activity, the presence of mtDNA-encoded COX subunit 2, and, more indirectly, the quality of the mitochondrial membrane potential. COX activity in individual mitochondria was also studied by electron microscopic cytochemistry. As a cellular model, we chose cybrid clones with various proportions of the MELAS mutation (A3243G) involving the mtDNA leucine tRNA gene. MELAS is an acronym for mitochondrial encephalopathy, lactic acidosis, and strokelike episodes. It describes a severe clinical syndrome among human mitochondrial diseases that was associated with the presence of the A3243G mutation of the mtDNA leucine tRNA gene in the vast majority of the patients that presented with the MELAS syndrome. The mutation was later associated with diverse clinical phenotypes such as isolated ophthalmoplegia or diabetes and deafness (12). The A3243G mutation is probably the most frequent mtDNA pathogenic point mutation in human pathology. Functional consequences of the mutation have been well studied in cybrid cells and are relatively mild, rendering relatively easy the analysis of cells with diverse mutation loads (14). Extensive direct complementation between wild-type and mutant mtDNA molecules has been demonstrated in these cells. The wild-type mtDNA threshold allowing a normal cell respiration rate was below $10 \%$ (14). We show that, in these cells, mitochondrial functions depending on the mtDNA were heterogeneous between cells spread out from clonal populations and between mitochondria from an individual cell. Intermitochondrial exchanges appeared, therefore, unable to distribute the complementing molecules evenly in the whole cell.

\section{METHODS}

Cybrid clones. Cybrids clones were a generous gift from Dr. E.A. Shoubridge (Montreal Neurologic Institute, Quebec, Canada). They were obtained by fusing rho0 cells from the osteosarcoma cell line 143B.TK ${ }^{-}$with cytoplasts from clonal pri-

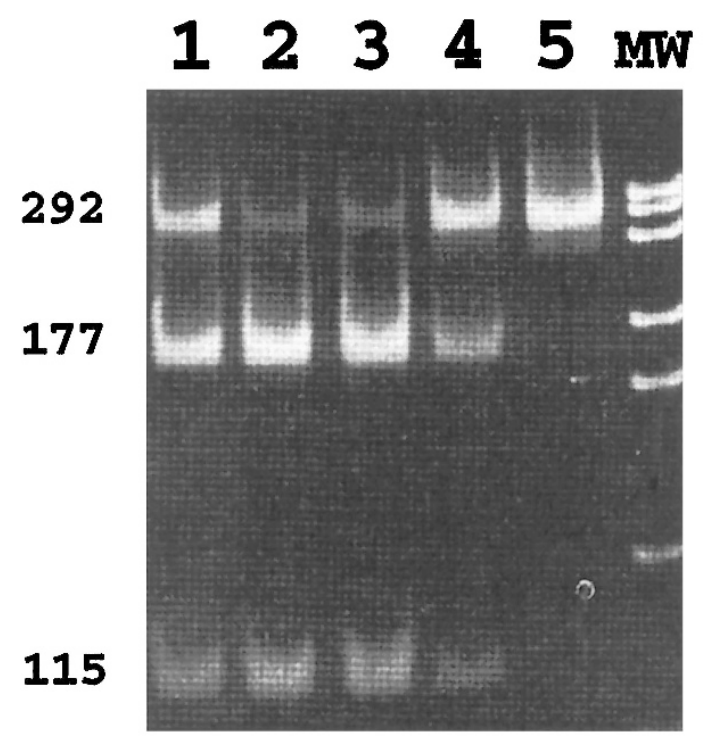

Figure 1. Quantitation of mutant mtDNA using ApaI restriction. Five percent acrylamide gel stained with ethidium bromide. $1-5$, Clone with 50, 95, 90, 35, and $0 \%$ mutation proportion, respectively. $M W$, Molecular weight markers are $\lambda$ DNA HindIII and $\Phi$ X174 HaeIII fragments. The presence of the A3243G mutation introduces a new restriction site that cleaves the 292 bp of the amplified mtDNA fragments in two fragments of 177 and $115 \mathrm{bp}$ (indicated in base pairs on the left side of the Figure). Radiolabeling during the last PCR cycle and quantitation of restricted radioactive fragments avoid underestimation of the mutant proportion due to the nondigestion of heteroduplex molecules. 


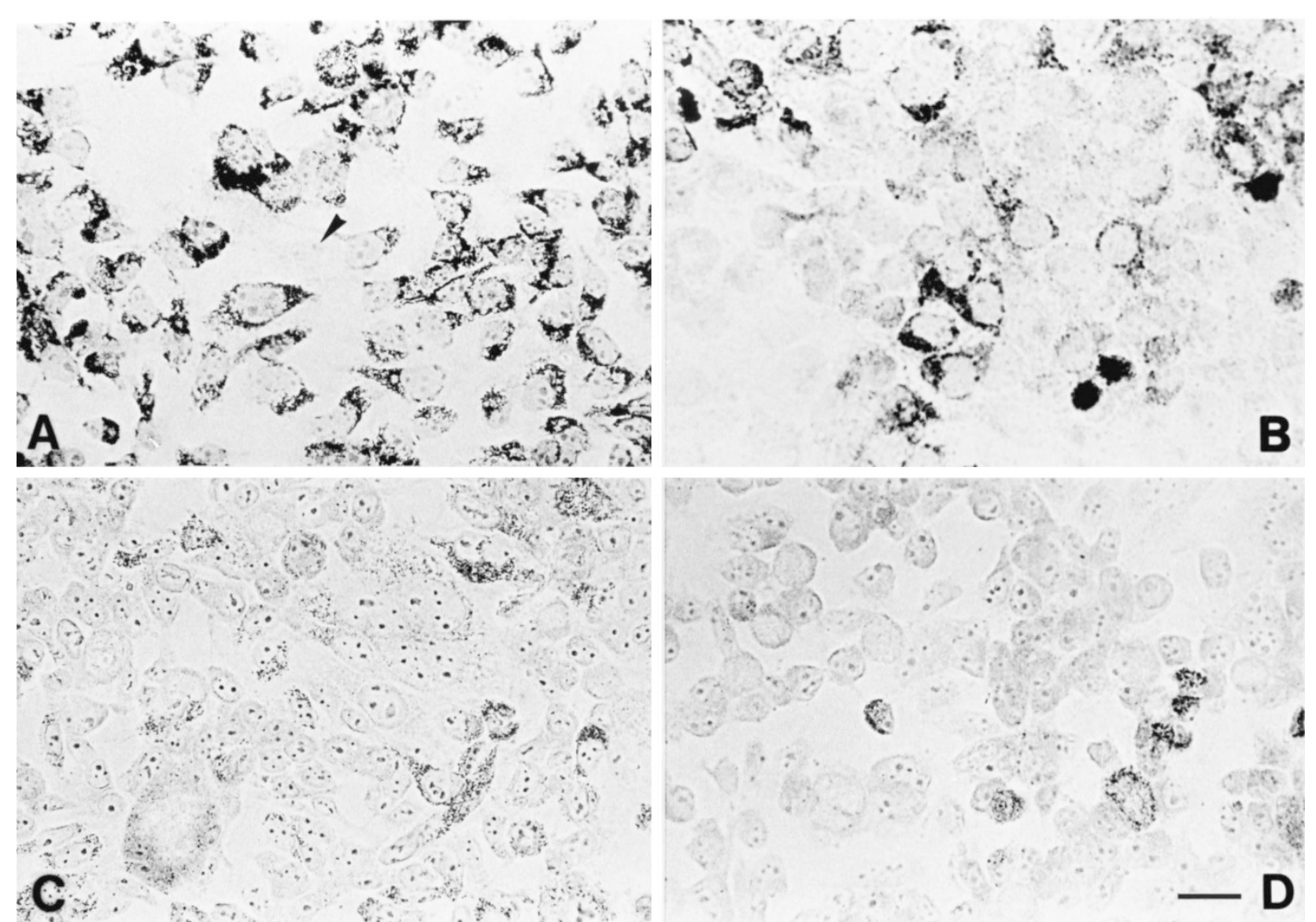

Figure 2. Histochemical analysis of COX activity. $(A)$ Clone with no detectable mutation, $(B) 35 \%$ mutation, $(C) 70 \%$ mutation, and $(D) 80 \%$ mutation. Arrowhead in $(A)$ points to a COX deficient cell. Bar $=10 \mu \mathrm{m}$.

mary myoblasts established from a patient carrying the A3243G mtDNA tRNA ${ }^{\text {leucine(UUR) }}$ point mutation (MELAS mutation) (15). Five different clones were cultured as described in DMEM enriched with $100 \mu \mathrm{g} / \mathrm{mL}$ pyruvate and $50 \mu \mathrm{g} / \mathrm{mL}$ uridine (15). Their initial mutation proportion was $0,55,80$, 96, and 99\%. MtDNA point mutation proportion was measured before each experiment on total cellular DNA by using ApaI restriction analysis of mtDNA amplified by the technique of the last radioactive PCR cycle (16). Uncut and digested fragments were counted with a $\beta$-imager. The proportion of the mutation was expressed as percent of total mtDNA.

\section{Histologic Analyses}

For histochemical and immunohistochemical analyses, 50,000 cells were plated on a coverslip, grown for $48 \mathrm{~h}$, washed with PBS pH 7.4, and air-dried for $20 \mathrm{~min}$.

COX histochemistry. Preincubation was performed at room temperature during $15 \mathrm{~min}$ in $50 \mathrm{mM}$ Tris- $\mathrm{HCl} \mathrm{pH} \mathrm{7.6,} 28$ $\mathrm{mg} / 100 \mathrm{~mL}$ cobalt chloride (CoCl2:6H2O), 0.5\% DMSO, and $10 \%$ sucrose. After one wash in $0.1 \mathrm{M}$ sodium phosphate $\mathrm{pH}$ $7.6,10 \%$ sucrose, cells were incubated during $6 \mathrm{~h}$ at $37^{\circ} \mathrm{C}$ in $0.1 \mathrm{M}$ sodium phosphate $\mathrm{pH} 7.6,10 \%$ sucrose, $1 \mathrm{mg} / \mathrm{mL}$ cytochrome $c$ type IV (Sigma Chemical Co.), $1 \mathrm{mg} / \mathrm{mL}$ DAB hydrochloride, $0.25 \% \mathrm{DMSO}$, and $20 \mu \mathrm{g} / \mathrm{mL}$ catalase. After another wash in $0.1 \mathrm{M}$ sodium phosphate $\mathrm{pH} 7.6,10 \%$ sucrose followed by PBS and distilled water, cells were mounted in warm gelatin and analyzed with optic microscopy. More than 400 cells were evaluated on black and white pictures to determine the fraction of COX-positive cells.

Succinate dehydrogenase (SDH) histochemistry. SDH histochemistry was performed as described (16).

Immunofluorescence of COX subunits: mtDNA-encoded subunit 2 (COX2) and nuclear DNA-encoded subunit 4 (COX4). Cells were preincubated $1 \mathrm{~h}$ at $37^{\circ} \mathrm{C}$ in $150 \mathrm{mM} \mathrm{NaCl}$, $25 \mathrm{mM}$ Tris $\mathrm{HCl} \mathrm{pH} 7.4$, and $0.01 \%$ saponin (TBSS) with 5\% normal goat serum. Incubation medium was TBSS with $1 \%$ BSA. Primary antibodies were rabbit polyclonal anti-COX2 and mouse monoclonal anti-COX4 (17). They were used together during $1 \mathrm{~h}$ at $37^{\circ} \mathrm{C}$ at a dilution of $1 / 200$ and $1 / 100$, respectively. Secondary antibodies were FITC-coupled antimouse and CY3-coupled anti-rabbit antibodies from Jackson (Jackson ImmunoResearch Laboratories, Inc., West Grove, PA, U.S.A.). They were used together during $1 \mathrm{~h}$ at $37^{\circ} \mathrm{C}$ at a dilution of $1 / 100$ and $1 / 200$, respectively. Images of the immunostained cells were acquired on a Leica TCS 4D confocal microscope, transferred to Adobe Photoshop (Adobe Systems Inc., Mountain View, CA, U.S.A.), and printed in an EPSON Stylus color 800 high-resolution printer (EPSON, France). More than 400 cells were evaluated on confocal images to determine the fraction of positive cells.

Mitochondrial membrane potential analysis. Fifty thousand cells were plated on a coverslip, grown for $48 \mathrm{~h}$, washed with 

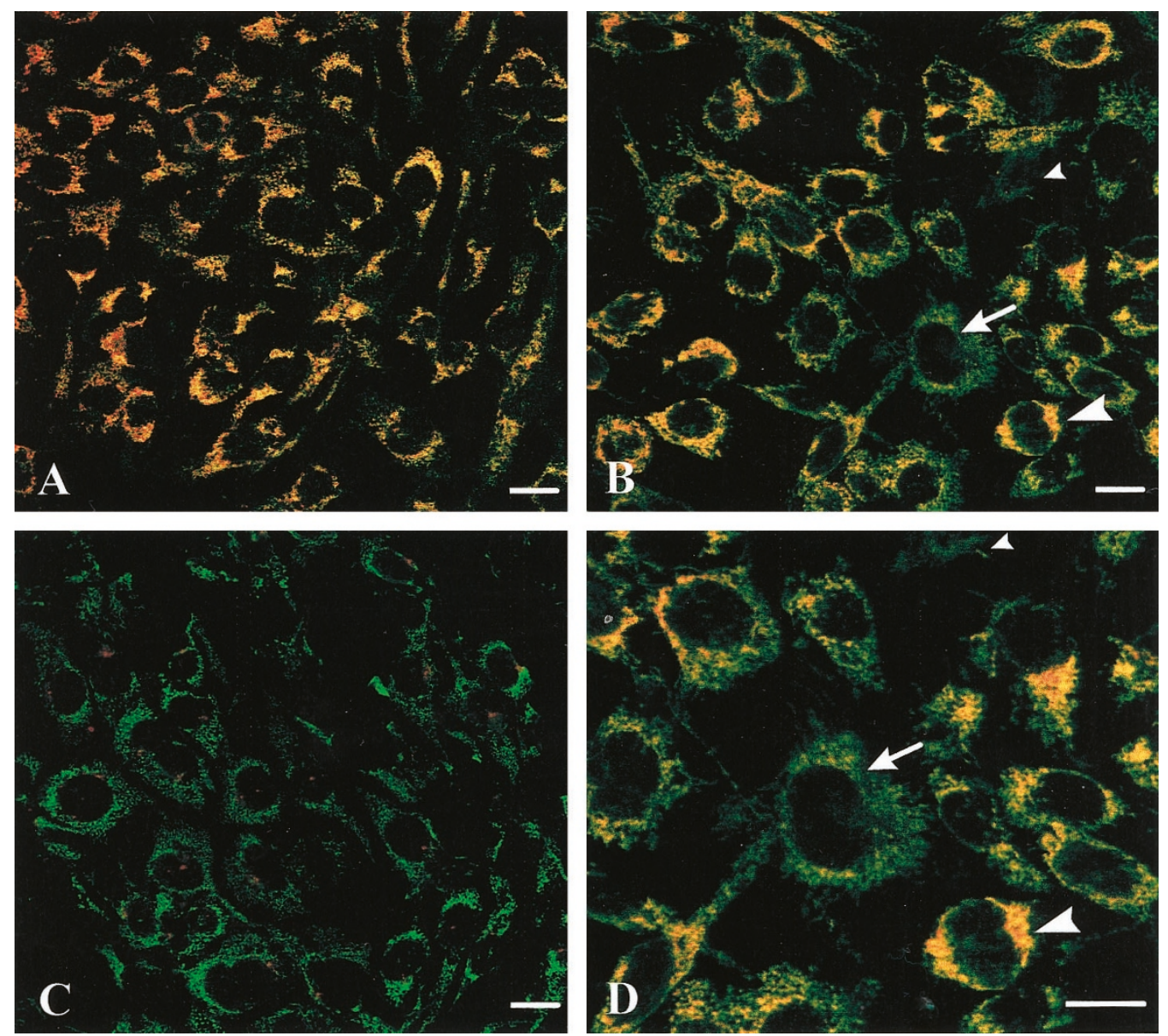

Figure 3. Immunofluorescent detection of two COX subunits: mtDNA-encoded COX subunit 2 (COX2, red signal) and nuclear DNA-encoded COX subunit 4 (COX4, green signal). Colocalization of both the green and red fluorescence signals in normal mitochondria gives a yellow signal. $(A)$ Merged confocal images of COX 2 and COX4 signals in a clone with no detectable mutation, $(B) 85 \%$ mutation, and $(C) 90 \%$ mutation. $(D)$ is a higher magnification of $(B)$. Bars $=5 \mu$ m.

PBS, and incubated for $30 \mathrm{~min}$ at $37^{\circ} \mathrm{C}$ in $5 \% \mathrm{CO}_{2}$ with 12 $\mu \mathrm{g} / \mathrm{mL} \mathrm{JC} 1$ in DMEM (18). After two washes in PBS, cells were mounted on microscope slides with a cavity and kept at $37^{\circ} \mathrm{C}$ until their analysis. The confocal images ( $\mathrm{Z}$ series) and quantitative analyses were performed as described for immunofluorescence.

\section{Ultrastructural Analyses}

One hundred thousand cells were plated on a coverslip and grown for $48 \mathrm{~h}$.

Morphology. Cells were treated at room temperature; they were washed with PBS, fixed in $2.5 \%$ glutaraldehyde in $0.1 \mathrm{M}$ sodium phosphate, $0.1 \mathrm{M}$ potassium phosphate $\mathrm{pH} 7.4$ for 15 min, washed with PBS, postfixed in $1 \%$ reduced osmium tetroxide in PBS, washed in PBS, washed in water, dehydrated in a graded series of acetone, and flat embedded at $60^{\circ} \mathrm{C}$ in Epon Polybed. Ultrathin sections $(70 \mathrm{~nm})$ were sectioned on a Leica Ultracut UCT (Vienna, Austria) with a diamond knife (Drukker, Holland), contrasted with uranyl acetate and lead citrate, and observed in a transmission electron microscope (Philips CM 120) operating at $80 \mathrm{kV}$.

COX cytochemistry. Cells were treated at room temperature; they were washed with PBS, fixed for $30 \mathrm{~min}$ in $2 \%$ paraformaldehyde in $\mathrm{PBS}$, and washed with $50 \mathrm{mM}$ Tris $\mathrm{HCl} \mathrm{pH} \mathrm{7.6,}$
$10 \%$ glucose. Incubation was performed as for COX histochemistry but during $3 \mathrm{~h}$ and without the preincubation step. After one wash in $50 \mathrm{mM}$ Tris $\mathrm{HCl} \mathrm{pH} \mathrm{7.6,10 \%} \mathrm{glucose,} \mathrm{cells}$ were treated as for ultrastructural morphology.

\section{RESULTS}

Using ApaI restriction, we measured the mutation proportion of the cell populations before each analysis (Fig. 1). In our culture conditions, the clones tended to lose their mutant mtDNA but at a very slow rate. The mean variation in mutant mtDNA proportion was $-0.8 \%$ per day with a range from -2.6 to $+2.9 \%$ on 22 measurements. Shift of heteroplasmy between cell plating and analysis (maximum $48 \mathrm{~h}$ ) was then negligible, and mutation proportion compared with observed histologic behavior of the cells.

As a relevant parameter of the mtDNA-dependent respiratory function, we analyzed COX histochemistry. This was performed on a total of 16 cell preparations from the five initial clones. Examples of clonal cell population without detectable mutation (Fig. $2 A$ ) are shown together with clones with 35 (Fig. 2B), 70 (Fig. 2C), and 80\% mutation proportion (Fig. $2 D)$. All preparations showed intercellular heterogeneity of COX activity. SDH histochemistry was performed as an internal control of nuclear-encoded cell respiratory functions. It 

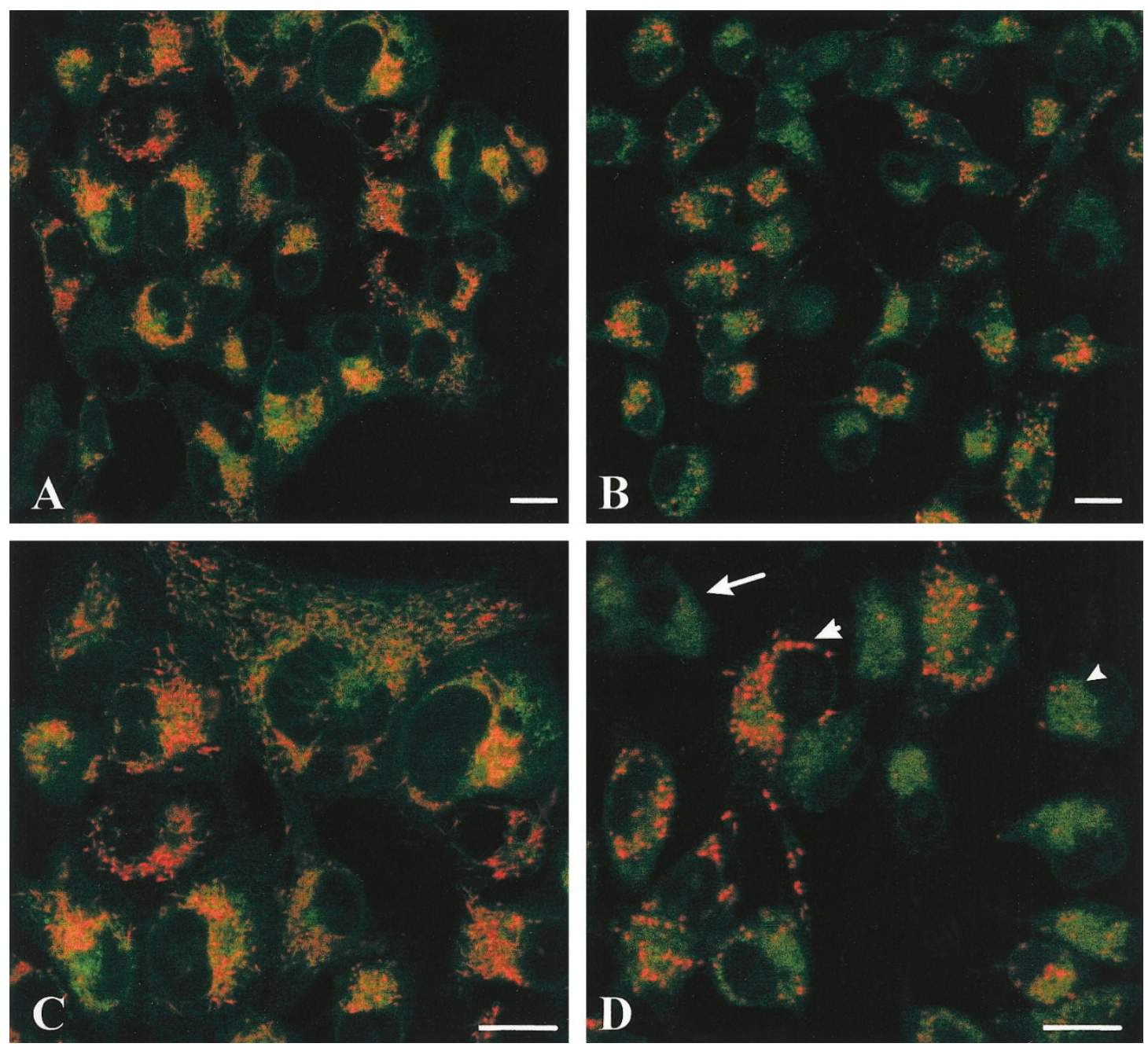

Figure 4. Mitochondrial membrane potential analysis using JC1. Merged confocal images after JC1 coloration $(A)$ in a clone containing no detectable mutation and $(B)$ in a clone with $90 \%$ mutation. Three cell types were defined: one normal type showing numerous brightly red J-aggregates (arrowhead), one intermediate type with less than five J-aggregates per cell (small arrowhead), and one deficient type without J-aggregates (arrow). $(C)$ and $(D)$ are higher magnification of $(A)$ and $(B)$, respectively. Bars $=5 \mu \mathrm{m}$.

showed an intense activity on the same cell preparations used for COX histochemistry, demonstrating that the observed reduction of COX activity was not due to a decrease in the number of mitochondrial organelles (not shown).

As the MELAS mutation involves an mtDNA tRNA gene, another relevant parameter to study was the presence of an mtDNA-encoded protein. We performed immunohistochemical detection of two COX subunits, one encoded by mtDNA (COX2) and the other by nuclear DNA (COX4). This was done on a total of 16 cell preparations from the five initial clones and shown in three representative clones (Fig. 3). Normal cells appeared as yellow due to the strict colocalization of both green and red fluorescent signals emitted by COX4 and COX2, respectively (Fig. 3A). Intercellular heterogeneity appeared only above the threshold of $80 \%$ mutation proportion. Normal cells coexisted with cells that showed a partial defect in COX2 with some yellow mitochondrial spots in a mostly green mitochondrial population as shown in Fig. $3 B$ (arrow) but also with cells with a profound defect in COX2 showing only green mitochondrial fluorescence (Fig. 3C). Intracellular mitochon- drial heterogeneity was shown on partially defective cells (arrow in Fig. 3D).

Maintenance of the mitochondrial membrane potential is linked to respiratory chain function but is not totally dependent upon its functionality, as, for example, mitochondrial membrane potential is partially maintained in rho 0 cells (10). This parameter was, therefore, interesting to study because it is more loosely coupled to mtDNA function. Furthermore, fixation artifacts may be avoided by the use of the vital lipophilic cation JC1 that aggregates in a potential-dependent manner. Aggregation of JC1 is accompanied by a shift of its fluorescence from green to red fluorescence, allowing direct evaluation of membrane potential (18). JC1 fluorescence was studied in four clones that had, respectively, 0, 62, 85, and 90\% mutation proportion at the time of the analysis (Fig. 4). Representative images were chosen from the clone without detectable mutation (Fig. 4A) and from the clone with the highest mutation load (Fig. 4B). Higher magnification of theses clones is shown in Figure 4, $C$ and $D$. Intercellular heterogeneity was obvious in all clones studied, and the images allowed us to 


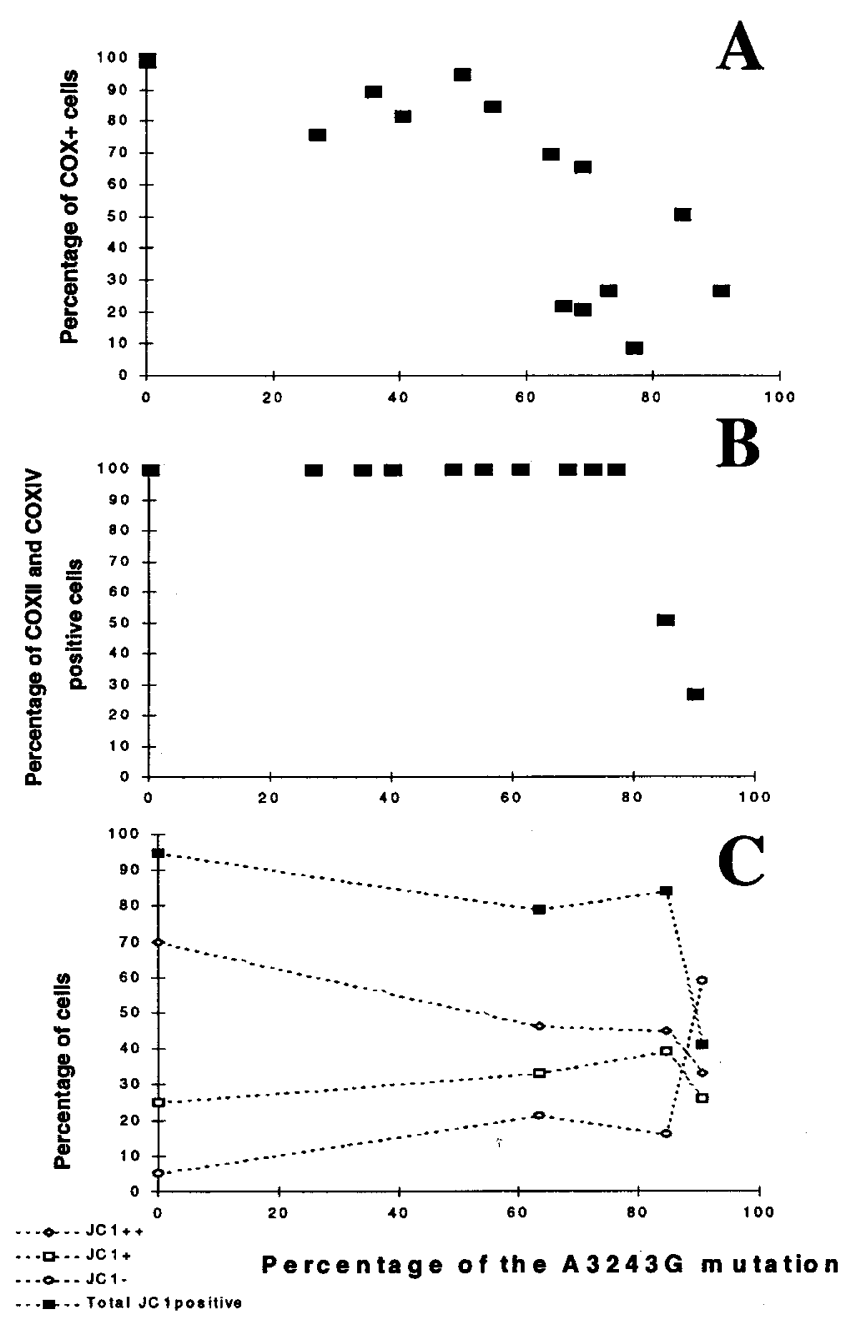

Figure 5. Correlation between mutation proportion and diverse mitochondrial characteristics. $(A)$ Percentage of cells with positive COX activity, $(B)$ percentage of cells with presence of both COX2 and COX4 fluorescent signals as shown in Figure 3, $(C)$ percentage of the different cell types with respect to their mitochondrial membrane potential (as shown in Fig. 4): $J C 1++=$ cells with normal membrane potential shown by the presence of numerous brightly red J-aggregates, $\mathrm{JCl}+=$ intermediate cell type characterized by the presence of a reduced number of J-aggregates $(<5$ per cell), $J C l-=$ deficient cells with only green mitochondria without visible J-aggregates, total $\mathrm{JC1}$ positive = sum of the $\mathrm{JC} 1+$ and $\mathrm{JC} 1++$ cells. Sum of the total $\mathrm{JC} 1$ positive cells and $\mathrm{JC} 1-$ cells $=100 \%$ of the cells.

distinguish three distinctive cell types: one with numerous brightly red aggregates (arrowhead, Fig. 4D), one lacking aggregates (arrow, Fig. 4D), and one with less than five aggregate clusters per cell (small arrowhead, Fig. 4D). Intracellular heterogeneity was obvious in cells with less than five aggregates in which few mitochondria emitted bright red fluorescence among a majority of mitochondria emitting only a green signal.

The proportion of deficient cells appeared to roughly follow that of mutant mtDNA molecules for the three characteristics that were analyzed but with different apparent thresholds (Fig. 5). Cells defective in their COX activity were noted above $50 \%$ mutation proportion (Fig. 5A) when cells defective in their mtDNA-encoded COX2 subunit were present only above $80 \%$ mutation proportion (Fig. 5B). Variations in the mitochondrial membrane potential were noted above $60 \%$ mutation proportion, the number of cells with numerous aggregates decreased, and that with less than five aggregate clusters increased, but there was very little increase of cells lacking aggregates up to 90\% mutation proportion (Fig. 5C).

Individual mitochondria COX activity was analyzed in four clones using equivalent cell density, plating duration, and the same fixing and staining solutions. The clones had, respectively, $0,52,77$, and $84 \%$ mutation proportion at the time of the analysis. Intracellular heterogeneity of individual mitochondria COX activity was evidenced in all the clones with coexistence of COX active (with DAB precipitate) and COXdeficient mitochondria (Fig. 6). COX-deficient mitochondria were often difficult to recognize and hampered the exact evaluation of their proportion. However, ultrastructural morphologic analysis of the clones had shown a similar number of mitochondria in the four clones (data not shown), whereas the number of COX active mitochondria clearly decreased with increasing mutation proportion. Deficient mitochondria were very rare in the clone with no detectable mutation (arrow, Fig. $6 A$ ). In the cells from the clone with a mutant load of $52 \%$, only a few mitochondria showed a COX defect with altered morphology, swelling and a disruption of the cristae (arrows and arrowhead, Fig. 5B). Other mitochondria in these cells showed COX activity but with a lower density of DAB precipitate density than in mitochondria from the clone without detectable mutation when both clones were incubated in parallel experiments. In the two clones with the highest mutation proportion, COX-defective mitochondria represented the majority of the mitochondria (Fig. 5, $C$ and $D$ ), but the distribution of the COX defect appeared different. In the clone with $77 \%$ mutant load, very few mitochondria showed COX activity (arrowheads, Fig. $6 C$ ), whereas a vast majority of them appeared as totally defective and morphologically altered (arrows, Fig. $6 C$ ). In the clone with $84 \%$ mutant load, most mitochondria showed some COX activity but only in a small part of the organelle (arrows, Fig. $6 D)$.

\section{DISCUSSION}

We chose to use histologic techniques because they allow us to analyze, in parallel experiments, many complementary aspects of the mitochondrial functions on a relatively small number of cells. Our results show that individual cells derived from heteroplasmic clonal cell populations are heterogeneous with respect to their mtDNA-dependent mitochondrial functions: COX activity, steady state amount of mtDNA-encoded COX2 subunit, and mitochondrial membrane potential. This intercellular phenotype heterogeneity is interpreted as the result of stochastic mitotic segregation of heterogeneous intracellular mitochondrial populations. Indeed, intracellular mitochondrial heterogeneity was directly seen during subcellular analysis of the mitochondrial membrane potential and COX2 subunit expression by confocal microscopy. Such direct visualization was impossible with light microscopy used in COX histochemistry. However, cells with positive but reduced COX histochemical activity were present in clones that appeared totally normal with SDH histochemical analysis. This could be 

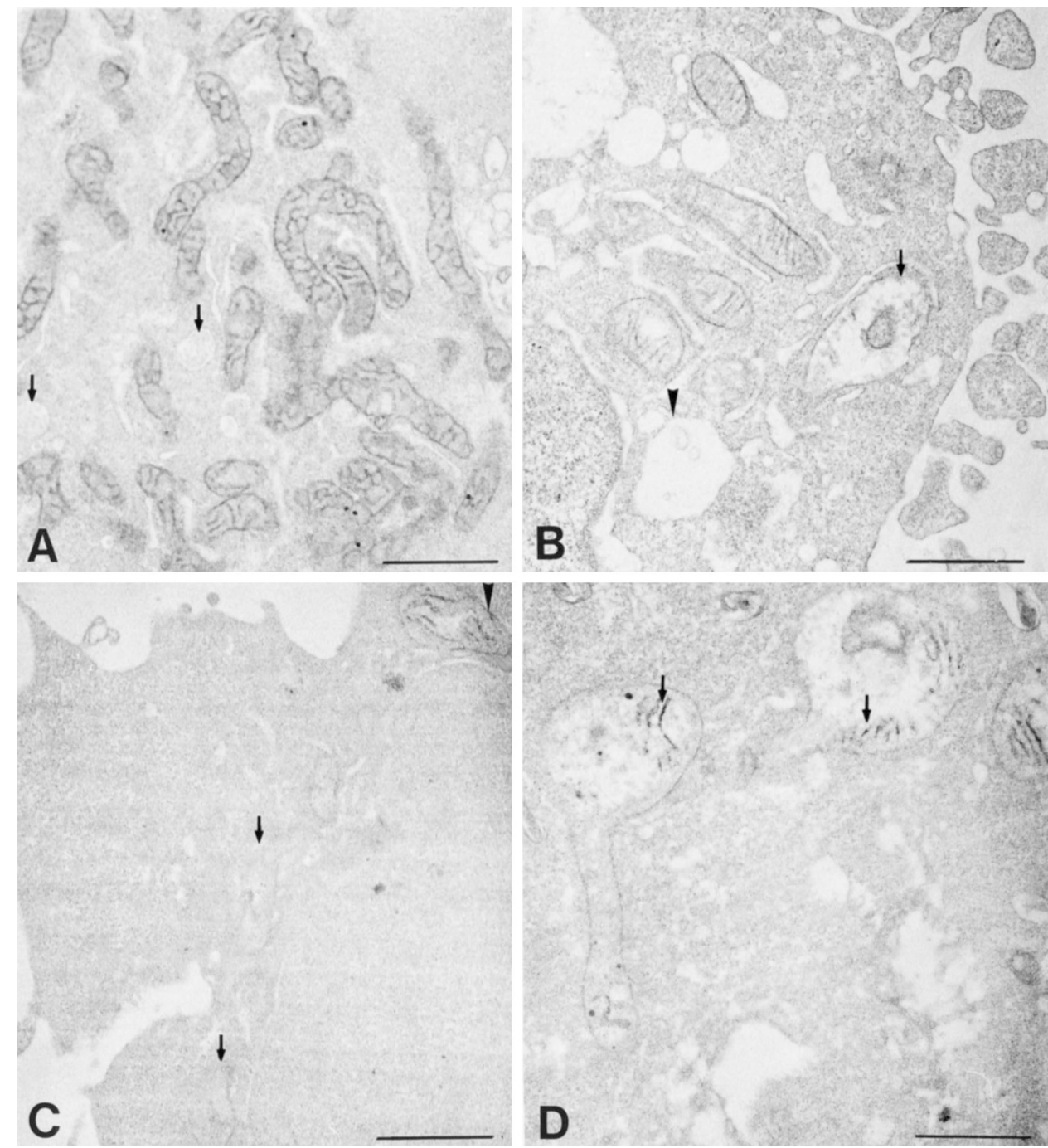

Figure 6. Ultrastructural analysis of COX activity using cytochemistry. $(A)$ Clone with no detectable mutation, $(B)$ with $52 \%$ mutation, $(C)$ with $77 \%$ mutation, and $(D)$ with $84 \%$ mutation. In $(A)$, most mitochondria show normal morphology and COX labeling; few mitochondria with abnormal morphology and defective COX activity are detected. In (B), mitochondria with defective COX activity and altered morphology are indicated by an arrow (swelling) and an arrowhead (cristae disruption). In $(C)$, the arrowhead points to a COX-positive mitochondrion, and arrows point to mitochondria without detectable COX activity. In (D), arrows point to the few COX-positive cristae in swollen mitochondria. Bars $=1 \mu \mathrm{m}$

interpreted as evidence for the presence of SDH-positive/COXnegative mitochondria in these cells and, therefore, as indicative of intracellular mitochondrial heterogeneity. Ultrastructural analysis confirmed COX activity heterogeneity among different mitochondria from the same cell. The diversity of the analytic procedures (histochemistry, immunohistochemistry, living cell staining, and cytochemistry) allowed us to vary the fixation procedures and, therefore, their possible artifacts. The number of defective cells in their mtDNA-dependent parameters followed roughly the mutant load (Fig. 5), thus implying that their defect was due to the mtDNA mutation. This was strengthened by the concomitant preservation of SDH activity or COX4 subunit steady state amount that does not depend on the mtDNA.

In most of our experiments, there was a mutant mtDNA threshold below which no defect could be observed. Previous estimations of this complementation threshold have been obtained at the cell population level by biochemical techniques such as polarography $(14,19)$. It was significantly higher than the threshold that we see using histologic analyses of complementation, because individual deficient cells are easily detected when their impact on the whole cell population would be ignored. The observed threshold level depended, however, on the analyzed parameter. Detection efficiency varies greatly among techniques and explains part of this discrepancy. COX histochemistry has a low sensitivity. Cells will, therefore, appear as deficient even in the presence of a significant residual activity. On the contrary, fluorescence detection is very sensitive, and cells with a partial decrease of the protein will give a still strong signal that will be difficult to differentiate from normal. On the other hand, the mutant mtDNA threshold may also reflect true differences in the mtDNA-dependence of different mitochondrial functions. COX activity and COX subunit 2 steady state level were chosen because they appeared as sensitive indicators of mtDNA translation defects in the investigation of human mitochondrial disorders $(16,20)$. COX catalytic subunits (COX1, 2, and 3) are encoded on mtDNA, and they have high transcription and translation steady states $(21,22)$. This 
probably explains the high sensitivity of both COX activity and COX2 subunit to mtDNA alterations. Mitochondrial membrane potential is less sensitive to mtDNA alterations.

The rapid segregation of heterogeneous cells from clonal populations that we observed contrasted with the relative stability of the heteroplasmy measured at the level of the whole cell population. This discrepancy is well explained by the highly permissive conditions of culture that allowed a normal growth curve even for severely respiratory-deficient cells.

The possibility of intermitochondrial complementation has been recently demonstrated using cybrids in which two pathogenic mutations were introduced separately $(5,11)$. In these experiments, however, intermitochondrial molecular exchanges appeared as very slow and/or very inefficient because of the very low number of complementing clones (5), the necessity to use highly permissive culture conditions to obtain them (11), and the presence of a roughly equal amount of the two complementing mtDNA populations (11). In the situation encountered in human pathology, wild-type and mutant mtDNA coexisted since the mutation event. In this situation, complementing cybrid clones are relatively easy to obtain even under selective culture conditions, and complementation occurs in the presence of very low proportions of wild-type mtDNA. Our histologic approach allowed us, however, to show that even in that latter situation, intermitochondrial molecular exchanges cannot distribute complementing molecules such as the wild-type mtDNA, tRNA, or proteins evenly to the whole cell. Diffusion of molecules between mitochondria appears, therefore, as the likely determining factor underlying most of the experimental controversies $(5,8,10,11)$ among which the differences in complementation efficiency with the initial localization of the complementing mtDNA molecules. Diffusion characteristics of mitochondrial components are beginning to be unraveled in yeast (23), but they are essentially unknown in humans. Their determination deserves, however, our full attention not only because the exchange of components between mitochondrial deals with a fundamental issue in cell biology but also because of potential consequences regarding therapeutic approaches to human diseases associated with heteroplasmic mtDNA mutations.

The osteosarcoma cybrid clones used in the present study represent a very useful model to study mitochondrial genetics. However, whether their mitochondrial compartment behavior is representative of that of other human cells remains disputable. Similar analyses of other human cell types are needed to assess this point.

\section{REFERENCES}

1. Attardi G, Schatz G 1988 Biogenesis of mitochondria. Ann Rev Cell Biol 4:289-333
2. Zeviani M, Fernandez-Silva P, Tiranti V 1997 Disorders of mitochondria and related metabolism. Curr Opin Neurol 10:160-167

3. Lightowlers RN, Chinnery PF, Turnbull DM, Howell N 1997 Mammalian mitochondrial genetics: heredity, heteroplasmy, and disease. Trends Genet 13:450-455

4. Hayashi JI, Ohta S, Kikuchi A, Takemitsu M, Goto Y, Nonaka I 1991 Introduction of disease-related mitochondrial DNA deletions into HeLa cells lacking mitochondrial DNA results in mitochondrial dysfunction. Proc Natl Acad Sci USA 88:10614-10618

5. Takai D, Inoue K, Goto Y, Nonaka I, Hayashi JI 1997 The interorganellar interaction between distinct human mitochondria with deletion mutant mtDNA from a patient with mitochondrial disease and with HeLa mtDNA. J Biol Chem 272:6028-6033

6. White FA, Bunn CL 1984 Segregation of mitochondrial DNA in human somatic cell hybrids. Mol Gen Genet 197:453-460

7. Jenuth JP, Peterson AC, Fu K, Shoubridge EA 1996 Random genetic drift in the female germline explains the rapid segregation of mammalian mitochondrial DNA. Nat Genet 14:146-151

8. Yoneda M, Miyatake T, Attardi G 1994 Complementation of mutant and wild-type human mitochondrial DNAs coexisting since the mutation event and lack of complementation of DNAs introduced separately into a cell within distinct organelles. Mol Cell Biol 14:2699-2712

9. Oliver N, Wallace DC 1982 Assignment of two mitochondrially synthesized polypeptides to human mitochondrial DNA and their use in the study of intracellular mitochondrial interaction. Mol Cell Biol 2:30-41

10. Hayashi JI, Takemitsu M, Goto Y, Nonaka I 1994 Human mitochondria and mitochondrial genome function as a single dynamic cellular unit. J Cell Biol 125:43-50

11. Takai D, Isobe K, Hayashi J 2021999 Transcomplementation between different types of respiration-deficient mitochondria with different pathogenic mutant mitochondrial DNAs. J Biol Chem 274:11199-11202

12. Chinnery PF, Howell N, Lightowlers RN, Turnbull DM 1997 Molecular pathology of MELAS and MERRF. The relationship between mutation load and clinical phenotypes. Brain 120:1713-1721

13. Taylor RW, Chinnery PF, Turnbull DM, Lightowlers RN 1997 Selective inhibition of mutant human mitochondrial DNA replication in vitro by peptide nucleic acids. Nat Genet 15:212-215

14. Chomyn A, Martinuzzi A, Yoneda M, Daga A, Hurko O, Johns D, Lai ST, Nonaka I, Angelini C, Attardi G 1992 MELAS mutation in mtDNA binding site for transcription termination factor causes defects in protein synthesis and in respiration but no change in levels of upstream and downstream mature transcripts. Proc Natl Acad Sci USA 89:4221-4225

15. King MP, Attardi G 1989 Human cells lacking mtDNA: repopulation with exogenous mitochondria by complementation. Science 246:500-503

16. Moraes CT, Ricci E, Bonilla E, DiMauro S, Schon EA 1992 The mitochondrial tRNA Leu(UUR) mutation in mitochondrial encephalomyopathy, lactic acidosis, and strokelike episodes (MELAS): genetic, biochemical, and morphological correlations in skeletal muscle. Am J Hum Genet 50:934-949

17. Possekel S, Lombès A, Ogier de Baulny H, Cheval MA, Fardeau M, Kadenbach B, Romero BN 1995 Immunohistochemical analysis of muscle cytochrome $c$ oxidase deficiency in children. Histochemistry 103:59-68

18. Smiley ST, Reers M, Mottola-Hartshorn C, Lin M, Chen A, Smith TW, Steele GDJ, Chen LB 1991 Intracellular heterogeneity in mitochondrial membrane potentials revealed by J-aggregate-forming lipophilic cation JC-1. Proc Natl Acad Sci USA 88:3671-3675

19. King MP, Koga Y, Davidson M, Schon EA 1992 Defects in mitochondrial protein synthesis and respiratory chain activity segregate with the tRNA Leu (UUR) mutation associated with mitochondrial myopathy, encephalopathy, lactic acidosis, and strokelike episodes. Mol Cell Biol 12:480-490

20. Lombès A, Mendell JR, Nakase H, Barohn RJ, Bonilla E, Zeviani M, Yates AJ, Omerza J, Gales TL, Nakahara K 1989 Myoclonic epilepsy and ragged-red fibers with cytochrome $c$ oxidase deficiency: neuropathology, biochemistry, and molecular genetics. Ann Neurol 26:20-33

21. Lightowlers R, Chrzanowska-Lightowlers Z, Marusich M, Capaldi RA 1991 Subunit function in eukaryote cytochrome $c$ oxidase. J Biol Chem 266:7688-7693

22. Attardi G, Cantatore P, Chomyn A, Crews S, Gelfand R, Merkel C, Montoya J, Ojala D 1982 A comprehensive view of mitochondrial gene expression in human cells. In: Slonimski P, Borst P, Attardi G (eds) Mitochondrial Genes. Cold Spring Harbor Laboratory, Cold Spring Harbor, NY, pp 51-71

23. Okamoto K, Perlman PS, Butow RA 1998 The sorting of mitochondrial DNA and mitochondrial proteins in zygotes: preferential transmission of mitochondrial DNA to the medial bud. J Cell Biol 142:613-623 